EPJ Web of Conferences 31, 00008 (2012)

DOI: $10.1051 /$ epjconf/20123100008

(C) Owned by the authors, published by EDP Sciences - SIF, 2012

\title{
An interpretation of staggering effects by correlation observables
}

M. D'Agostino ${ }^{1}$, M. Bruno ${ }^{1}$, F. Gulminelli $^{2}$, L. Morelli ${ }^{1}$, G. Baiocco ${ }^{1,2}$, L. Bardelli ${ }^{3}$, S. Barlini ${ }^{3}$, F. Cannata ${ }^{1}$, G. Casini $^{3}$, E. Geraci ${ }^{4}$, F. Gramegna ${ }^{5}$, V. L. KravchuK ${ }^{5}$, T. Marchi ${ }^{5,6}$, A. Moroni ${ }^{7}$, A. Ordine ${ }^{8}$ and Ad. R. Raduta ${ }^{9}$

1 Dipartimento di Fisica dell'Università and INFN, Bologna, Italy

2 CNRS, UMR6534, LPC ,F-14050 Caen cédex, France and ENSICAEN,

UMR6534, LPC, F-14050 Caen cédex, France

3 INFN, Firenze, Italy

4 Dipartimento di Fisica dell'Università and INFN, Catania, Italy

${ }^{5}$ INFN, Laboratori Nazionali di Legnaro, Italy

6 Dipartimento di Fisica dell'Università, Padova, Italy

7 INFN, Milano, Italy

8 INFN, Napoli, Italy

9 NIPNE, Bucharest-Măgurele, POB-MG6, Romania

\begin{abstract}
The reactions ${ }^{32} S+{ }^{58,64} N i$ are studied at $14.5 \mathrm{~A} \mathrm{MeV}$. Evidence is found for odd-even effects in isotopic observables of the decay of a projectile-like source. The influence of secondary decays on the staggering is studied with a correlation function technique, showing that odd-even effects are due to interplay between pairing effects in the nuclear masses and in the level densities.
\end{abstract}

\section{Introduction}

Experimental studies of odd-even effects in fragment production have been performed since a long time. Recent experiments performed with $4 \pi$ detectors have added experimental information on these anomalies [1-3]. However

This is an Open Access article distributed under the terms of the Creative Commons Attribution License 2.0, which permits unrestricted use, distribution, and reproduction in any medium, provided the original work is properly cited. 
all these results have not produced clear-cut conclusions about their interpretation. A priori, these effects point to the pairing residual interaction and its dependence on temperature. Getting experimental information on this issue is of importance both in nuclear physics and in astrophysics [4]. Understanding the origin of odd-even effects is also relevant for studies on symmetry energy, which can be linked to the isotopic distributions if these latter are not too much perturbed by secondary decays [5].

In recent papers [3], we have reported on an experimental study of staggering in $\mathrm{S}+\mathrm{Ni}$ collisions at $14.5 \mathrm{~A} \mathrm{MeV}$. Important odd-even effects have been observed in peripheral and central collisions. A detailed study of the most probable decays contributing to the yield of the different isotopic chains has additionally indicated that the lowest emission threshold for particle production cannot be the unique factor governing the staggering.

To quantitatively control the population at the last-but-one evaporation step, we present here an experimental estimate of secondary evaporation using a correlation function based technique. We show that odd-even effects are already present in fragment yields excited above the particle emission threshold, indicating that both pairing effects on the nuclear masses and on the level densities should be considered to explain these oscillations.

\section{Experimental distributions}

The reactions ${ }^{32} S+{ }^{58,64} \mathrm{Ni}$ at $14.5 \mathrm{AMeV}$ were measured at the TANDEMALPI acceleration system of the Legnaro National Laboratory, with the hight acceptance detecting device GARFIELD coupled to an annular threestage detector (Ring Counter) [6]. The sorting of the measured events as a function of the centrality has been performed with the method of the "shape analysis" [3]. A comparison to the GEMINI evaporation code suggests that peripheral events are associated to the evaporation from a quasi-projectile source with $\left\langle E^{*}>\approx 1 \mathrm{~A} \mathrm{MeV},\langle Z>\approx 16,\langle A>\approx 32\right.$, and an angular momentum $J=0 \div 16 \hbar$.

Odd-even effects in the elemental fragment charge distribution are clearly established (see Fig. 1) and can be highlightened by the ratio of the measured distribution and its smoothing, obtained by a parabolic interpolation of the measured yields over 5 consecutive points. Considering that for almost all the isotopically resolved yields $(Z \leq 8)$ the most abundant isotope of each element is $N=Z$ (see Fig. 7 of Ref. [3]), the observed staggering can be interpreted as dominance of even-even isotopes over odd-odd ones. This can be easily understood [2] from the pairing contribution to the isotope masses, 

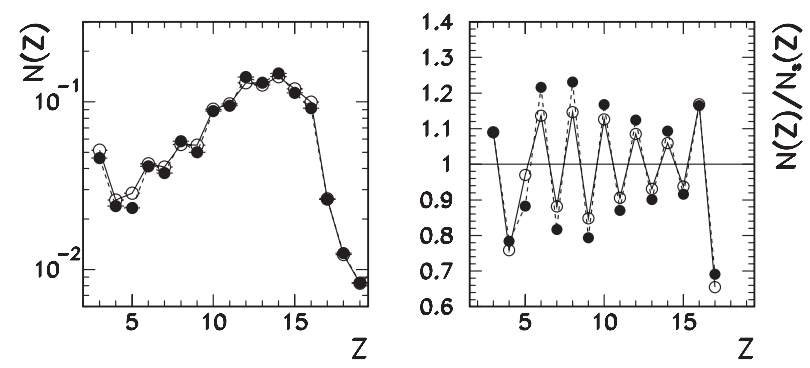

Figure 1: Left part: elemental fragment $(Z \geq 3)$ distribution for the QuasiProjectile fragments in ${ }^{32} S+{ }^{58} \mathrm{Ni}$ (full symbols, dashed line) and ${ }^{32} \mathrm{~S}+{ }^{64} \mathrm{Ni}$ (open symbols, full line). Right part: Ratio of the measured by the smoothed distributions. Lines are drawn to guide the eye.

if the last evaporation step corresponds to nucleon emission. However, since for the $N=Z$ isotopes the lowest emission threshold typically corresponds to $\alpha$ decay and the $\alpha$ separation energy shows a smooth behavior, the extra yield of even-even isotopes is not explained by the energy balance of the last evaporation step. It is then necessary to recover experimental information on the population of parent nuclei prior to their last decay by particle emission.

\section{Warm fragments from correlation functions}

For a quantitative understanding of odd-even effects we propose a backtracing technique based on correlation functions of the relative kinetic energy of isotope pairs. If we concentrate on light nuclei $(3 \leq Z \leq 8)$, their discrete spectrum is so extended that the last particle evaporation step takes place typically from a discrete resonance, which can at least in principle be recognized as a peak in a relative kinetic energy two-body correlation function. Experimentally the two-particle correlation function is defined as:

$$
\sum_{\left(\overrightarrow{p_{1}}-\overrightarrow{p_{2}}\right)^{2} / 2 \mu=E_{r e l}} Y_{12}\left(\overrightarrow{p_{1}}, \overrightarrow{p_{2}}\right)=C\left[1+R\left(E_{r e l}\right)\right] \cdot \sum_{\left(\overrightarrow{p_{1}}-\overrightarrow{p_{2}}\right)^{2} / 2 \mu=E_{r e l}} Y_{1}\left(\overrightarrow{p_{1}}\right) Y_{2}\left(\overrightarrow{p_{2}}\right)
$$

where $Y_{12}$ is the two-particle coincidence yield of a given pair of particles with momenta $\vec{p}_{1}$ and $\vec{p}_{2}$, and the $Y_{i}\left(\vec{p}_{i}\right)$ are the single particle yields for two particles belonging to different events. The summations of eq. 1 run over pairs of momenta $\vec{p}_{1}$ and $\vec{p}_{2}$ corresponding to the same bin in relative energy $E_{r e l}$. The correlation function describes how the correlation between interacting particles measured in the same event differs from the underlying two-particle phase space, modeled by the mixing event technique [8]. The constant $C$ is the ratio of the total numbers of mixed and coincident pairs. 


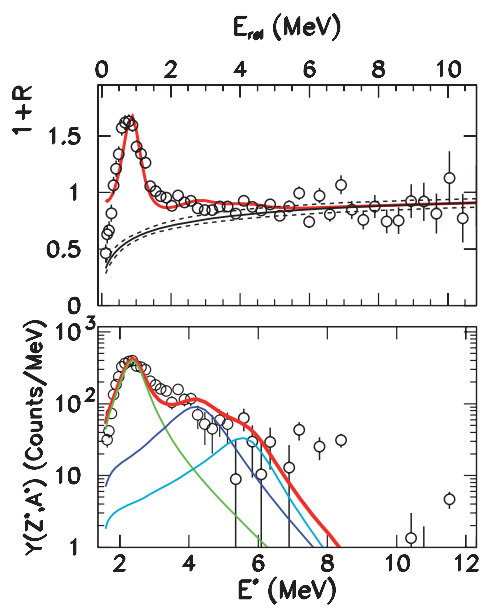

Figure 2: (Color online) Upper part: relative kinetic energy correlation function (symbols) of $d-\alpha$ pairs measured in peripheral ${ }^{32} S+{ }^{58} N i$ collisions, fitted through Eq. 4 (thick solid line). The Coulomb background (thin solid line) is indicated together with its uncertainties (dashed lines). Lower part: experimental population of primary ${ }^{6} L i^{*}$ parents (symbols) and single excited state contributions (thin lines) together with their sum (thick line) as a function of the excitation energy: $E^{*}=$ $E_{r e l}+Q_{V}$, with $Q_{V}=\mathrm{Q}$-value of the decay.

To investigate the decay of particle unbound states one has to disentangle the long range Coulomb and short range nuclear contributions to the two particle phase space:

$$
1+R\left(E_{\text {rel }}\right)=1+R_{\text {Coul }}\left(E_{\text {rel }}\right)+R_{\text {nuc }}\left(E_{\text {rel }}\right),
$$

and perform a fit of the measured correlations, by empirically parametrizing the Coulomb contribution [8]:

$$
1+R_{\text {Coul }}\left(E_{\text {rel }}\right)=1-\exp \left[-\left(E_{\text {rel }} / E_{c}\right)^{\gamma}\right]
$$

and the nuclear part:

$R_{n u c}\left(E_{r e l}\right)=\frac{e^{-E_{r e l} / T_{e f f}}}{\left(2 S_{1}+1\right)\left(2 S_{2}+1\right)} \frac{h^{3}}{4 \pi V_{f} \mu \sqrt{2 \mu E_{r e l}}} \frac{1}{\pi} \sum_{i} \frac{\left(2 J_{i}+1\right) \Gamma_{i} / 2(B . R .)}{\left(E_{r e l}-E_{i}^{*}\right)^{2}+\Gamma_{i}^{2} / 4}$

$S_{1}$ and $S_{2}$ are the spins of the considered particles, $\mu$ is their reduced mass, $V_{f}$ the effective emitting source volume, $T_{\text {eff }}$ the associated effective temperature, $J_{i}, E_{i}^{*}, \Gamma_{i}$ the spin, excitation energy and width of the level $i$, and (B.R.) is the branching ratio for decay to the measured channel. The free parameters of the fit are the Coulomb parameters $E_{c}, \gamma$ and $V_{f}, T_{e f f}$, which 
would represent a physical source volume and temperature only in the idealized situation of a single decay step of a fully equilibrated source. Only levels with spins, excitation energies, widths and branching ratios experimentally measured [7] were considered in the fit.

We report in Fig. 2 the $d-\alpha$ correlation function as an example of our analysis. More cases are reported in Ref. [3]. Primary yields, calculated by multiplying the nuclear contribution for the uncorrelated yield, $Y_{\text {cor }}\left(E^{*}\right)=$ $\left(R\left(E^{*}\right)-R_{\text {coul }}\left(E^{*}\right)\right) \sum_{E} Y_{1} Y_{2}$ [8], are shown by full symbols in the bottom panel of Fig. 2 together with the contributions from ${ }^{6} \mathrm{Li}^{*}$ excited levels entering in Eq. 4.

The total primary population of a given isotope at the last-but-one evaporation step $Y\left(A^{*}, Z^{*}\right)$ is calculated by numerically integrating the primary yields over the explored range of excitation energy. To evaluate the fraction
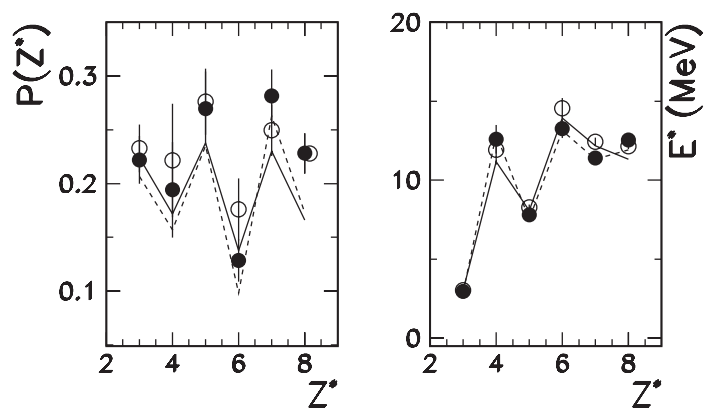

Figure 3: Population of primary fragments (left) and their average excitation energy (right) for the ${ }^{32} S+{ }^{58} N i$ (full symbols and dashed lines) and ${ }^{32} S+{ }^{64} \mathrm{Ni}$ (circles and full lines) peripheral data set.

of the coincident pairs emitted by "warm" pre-fragments, primary yields have been normalized to the sum of pairs having total charge equal to $Z^{*}$ : $P\left(Z^{*}\right)=\sum_{A^{*}} Y\left(A^{*}, Z^{*}\right) / \sum_{A^{*}} Y_{12}\left(A^{*}, Z^{*}\right)$. In Fig. 3 the filled $\left({ }^{32} S+{ }^{58} N i\right)$ and open $\left({ }^{32} S+{ }^{64} \mathrm{Ni}\right)$ circles are associated to the numerical integration of the estimated correlated yield $Y_{\text {cor }}$. The lines correspond to the integral of the fitting function Eq. $4 Y_{\text {res }}$, shown in the bottom of Fig. 2.

Reconstructed "warm" yields and average excitation energies (Fig. 3) keep on showing the staggering. Particularly striking is the fact that this staggering shows an opposite trend to the experimental asymptotic distributions shown in Fig. 1. This can be qualitatively understood as a level density effect: even-even nuclei have a lower level density at low energy because of the pairing gap, which leads to a reduced population at the last-but-one evaporation step. 
At least three decay channels have been measured for each parent $(3 \leq$ $Z^{*} \leq 8$ ), and many populated particle unstable levels contribute to the measured yield. This guarantees that no trivial auto-correlations between the $\mathrm{Z}$ of the daughter and the $\mathrm{Z}$ of the parent is at the origin of the behavior of the "warm" yields. Also efficiency corrections [3], evaluated by Monte Carlo calculations, do not modify the inverse staggering of primary warm isotopes.

As far as the lack of neutron detection is concerned, some hints come from GEMINI calculations. In figure 4 we present the calculated 'warm' elemental yields, obtained by summing up the cold products emitted in the last step by the same parent. Together with a complete reconstruction of the last-but-one evaporation step, we show the case similar to the experimental one, ignoring neutron decaying channels. The same staggering observed

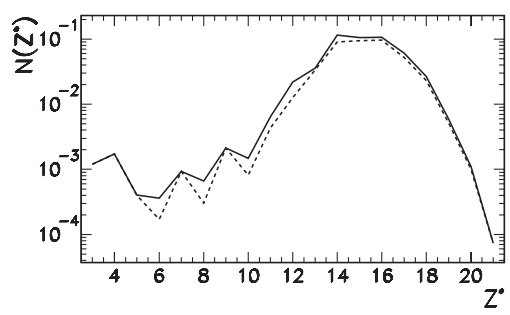

Figure 4: Elemental "warm" fragment $(Z \geq 3)$ distribution from the decay of a quasi projectile source, within the GEMINI evaporation model. Dashed line corresponds to a complete reconstruction of the last-but-one evaporation step, while the solid line is obtained ignoring neutron decaying channels.

for experimentally reconstructed primary fragments (Fig. 3) is present in GEMINI calculations, opposite to the trend of measured cold isotopes. This suggests that the 'warm' distribution at the last-but-one evaporation step is not qualitatively distorted by the lack of neutron detection.

\section{Conclusions}

We have reported on an experimental study of staggering in ${ }^{32} S+{ }^{58,64} \mathrm{Ni}$ collisions at $14.5 \mathrm{~A} \mathrm{MeV}$. Important odd-even effects are seen in the fragment yields produced in peripheral collisions. For the light isotopically resolved fragments, the discrete spectrum of excitation energy of "warm" prefragments extends over a wide energy range which can overcome the neutron and proton emission thresholds. This allows a partial reconstruction of the excited fragment yields prior to their last decay, through a model- 
independent correlation function technique.

The charge distribution of warm fragments displays odd-even effects opposite to the asymptotic distributions. This means that the quantitative understanding of the odd-even effect needs a global detailed control of the evaporation chain. Furthermore, the staggering cannot be only attributed to the pairing effect of nuclear masses, but is also influenced by pairing and isospin effects in the level density, thus being potentially useful to gather information of the temperature dependence of nuclear pairing.

\section{References}

[1] E.Geraci et al. Nucl.Phys. A732 (2004) 173; I.Lombardo et al. Phys.Rev. C84 (2011) 024613 and contribution to this conference; G.Ademard et al., Phys.Rev. C83 (2011) 054619; J.P.Wieleczko contribution to this conference; E.Galichet and M.F.Rivet, contribution to this conference.

[2] M.V.Ricciardi et al., Nucl.Phys. A733 (2004) 299; arXiv-1007.0386v1 and references quoted therein.

[3] M.D’Agostino et al., Nucl.Phys. A861 (2011) 47; Nucl.Phys.A doi:10.1016/j.nuclphysa.2011.11.011; L.Morelli contribution to this conference.

[4] A.Schiller et al., Phys.Rev. C63 (2001) 021306; N.Chamel et al., Phys.Rev. C81 (2010) 045804; A.Fantina et al., Phys.Lett. B676 (2009) 140 .

[5] M.Colonna and F.Matera, Phys.Rev. C71, 064605 (2005); Ad.R.Raduta and F.Gulminelli, Phys.Rev. C75 (2007) 044605; R.J.Charity et al. Phys.Rev. C63 (2001) 024611.

[6] F.Gramegna et al., Nucl.Instr. And Meth. A389 (1997) 474; A.Moroni et al., Nucl.Instr. And Meth. A556 (2006) 516.

[7] F.Gulminelli and D.Durand, Nucl.Phys. A615 (1997) 117; //www.nndc.bnl.gov/nudat2/

[8] J.Pochodzalla et al., Phys.Rev. C35 (1987) 1695; T.K.Nayak et al., Phys.Rev. C 45 (1992) 132; W.P.Tan et al. Phys.Rev. C69 (2004) 061304. 